\title{
Krankenhaushygiene aus Sicht der Hygienefachkräfte
}

\author{
Bernd Gruber
}

(C) Springer-Verlag 2012

Im Rahmen der stationären Behandlung sehen wir immer mehr Patienten, die mit multiresistenten Erregern besiedelt sind. Der Anstieg ist zum Beispiel bei der Klebsiella pneumonie mit Carbapenemase sehr deutlich. Hat das RobertKoch-Institut 2008 noch mitgeteilt, dass dieser Keim vereinzelt in Deutschland nachgewiesen wurde, finden wir ihn jetzt schon auf vielen Intensivstationen. Da wir nicht wissen, womit unsere Patienten bei Aufnahme besiedelt sind, ist die Basishygiene ein zwingendes Muss der Prävention. Bisher galt immer: a) Abstriche nehmen, b) Ergebnisse abwarten, und c) bei positiven Befunden Hygienemaßnahmen einleiten. Das bedeutet, dass zwei bis vier Tage lang keine Isolierungsmaßnahmen oder Sonstiges durchgeführt wurden und der Keim sich ungehindert durch die Stationen verteilen konnte.

Die Basishygienemaßnahmen für alle Mitarbeiter und Berufsgruppen sind 1) Händedesinfektion, 2) Handschuhe, 3) Mundnasenschutz und 4) Schutzschürze bzw. Schutzkittel, je nach Anamnese.

Antibiotikatherapien, Krankenhausaufenthalte oder chronische Wunden bei pflegebedürftigen Menschen haben einen erheblichen Einfluss auf die Keimbesiedlung.

Hygiene muss gelebt werden und zwar in allen Bereichen des Gesundheitswesens. Wir müssen uns der Verantwortung stellen, dass wir es sind, die die Keime verteilen. Die Entstehung ist häufig multifaktoriell, dies darf jedoch nicht als Ausrede benutzt werden. Allen Mitarbeitern muss klar sein, dass durch unsachgemäßes Handeln auch eine Eigengefährdung stattfinden kann. Es werden immer wieder Fälle beschrieben, bei denen Mitarbeiter aus dem Gesundheitswesen MRSA-Träger sind, die bei einem akutem Ereignis (Unfall, Notfall-OP) eigene Infektionen entwickeln.

B. Gruber $(\bowtie)$

Marienhospital Osnabrück, Osnabrück, Deutschland
Im Rahmen der Fehlerkultur in Deutschland muss es auch möglich sein, Kollegen auf Hygienefehler anzusprechen ohne Sanktionen vor allem durch Vorgesetzte zu erleiden. In der freien Wirtschaft, z. B. im Luftverkehr, wären Fehler durch Unterlassung nicht möglich. 